\title{
Data protection-compliant broad consent for secondary use of health care data and human biosamples for (bio)medical research: towards a new German national standard.
}

\author{
Sven Zenker ${ }^{*}{ }^{1}$, Daniel Strech ${ }^{\llbracket 2}$, Kristina Ihrig $^{3}$, Roland Jahns ${ }^{4}$, Gabriele Müller ${ }^{5}$, Christoph \\ Schickhardt ${ }^{6}$, Georg Schmidt ${ }^{7}$, Ronald Speer ${ }^{8}$, Eva Winkler ${ }^{9}$, Sebastian Graf von \\ Kielmansegg ${ }^{10}$, Johannes Drepper ${ }^{11}$
}

${ }^{1}$ Staff Unit for Scientific \& Medical Technology Development \& Coordination (MWTek), Commercial Directorate; Institute for Medical Biometry, Informatics \& Epidemiology; Department of Anesthesiology and Intensive Care Medicine, University Hospital Bonn, Venusbergcampus 1, 53127 Bonn, Germany

${ }^{2}$ QUEST Center, Berlin Institute of Health $(\mathrm{BIH})$ at Charité - Universitätsmedizin Berlin ${ }^{3}$ Department of Medicine, Hematology/Oncology, Goethe University, Theodor-Stern-Kai 7, 60590, Frankfurt am Main, Germany; German Cancer Consortium (DKTK), partner site Frankfurt/Mainz; German Cancer Research Center (DKFZ), Im Neuenheimer Feld 280, 69120 Heidelberg, Germany

${ }^{4}$ Interdisciplinary Bank of Biomaterials and Data Würzburg (ibdw), University and University Hospital of Würzburg, Building A8/A9, Straubmühlweg 2a, 97078 Würzburg, Germany,

${ }^{5}$ Center for Evidence-Based Healthcare, University Hospital Carl Gustav Carus and Carl Gustav Carus Faculty of Medicine, Technische Universität Dresden, Fetscherstr. 74, 01307 Dresden, Germany

${ }^{6}$ Section of Translational Medical Ethics, National Center for Tumor Diseases, German Cancer Research Center, Im Neuenheimer Feld 460, 69120 Heidelberg, Germany

${ }^{7}$ Department of Internal Medicine 1, Klinikum rechts der Isar, Technical University of Munich, Munich, Germany; German Centre for Cardiovascular Research partner site Munich Heart Alliance, Munich, Germany

${ }^{8}$ LIFE - Leipzig Research Center for Civilization Diseases, Medical Faculty, Leipzig University, Philipp-Rosenthal-Straße 27, 04103 Leipzig, Germany

${ }^{9}$ Section for Translational Medical Ethics, Dept Medical Oncology, National Center for Tumor Diseases, Heidelberg University Hospital, INF 460, 69121 Heidelberg

${ }^{10}$ Chair of Public Law and Medical Law, Kiel University, Leibnizstraße 2, 24118 Kiel, Germany

${ }^{11}$ TMF - Technology, Methods, and Infrastructure for Networked Medical Research, Charlottenstrasse 42, 10117 Berlin, Germany

${ }^{*}$ Corresponding author

Email: zenker@uni-bonn.de; ORCID ID https://orcid.org/0000-0003-0774-0725

IThese authors contributed equally to this work. 


\section{Abstract}

Background: The secondary use of deidentified but not anonymized patient data is a promising approach for enabling precision medicine and learning health care systems. In most national jurisdictions (e.g., in Europe and North America), this type of secondary use requires patient consent. While various ethical, legal, and technical analyses have stressed the opportunities and challenges for different types of consent over the past decade, no country has yet established a national consent standard accepted by the relevant authorities.

Methods: A working group of the national Medical Informatics Initiative in Germany conducted a requirements analysis and developed a GDPR-compliant broad consent standard involving all relevant stakeholder groups and authorities.

Results: This paper presents the broad consent text together with a guidance document on mandatory safeguards for broad consent implementation. The mandatory safeguards comprise i) independent review of individual research projects, ii) organizational measures to protect patients from involuntary disclosure of protected information, and iii) comprehensive information for patients and public transparency. This paper further describes the key issues discussed with the relevant authorities, especially the position on additional or alternative consent approaches such as dynamic consent.

Discussion: Both the resulting broad consent text and the national consensus process are relevant for similar activities internationally. A key challenge of aligning consent documents with the various stakeholders was explaining and justifying the decision to use broad consent and the decision against using alternative models such as dynamic consent. Public transparency for all secondary use projects and their results emerged as a key factor in this justification. While currently largely limited to academic medicine in Germany, the first steps for extending this broad consent approach to wider areas of application, including smaller institutions and medical practices, are currently under consideration. 


\section{Background}

The secondary use of routinely collected patient data $(R C D)$ such as electronic health records (EHRs) and human biosamples acquired in the health care context is a promising approach for enabling data-intensive biomedical research, precision medicine, and a learning health care system [1-4]. However, the implementation of an infrastructure for large-scale secondary use faces several technical, ethical, and legal challenges [5]. In Germany, as in other EU countries, the General Data Protection Regulation (GDPR), usually in combination with national laws (e.g., concerning medical confidentiality), defines the relevant data protection and privacy rules. Unfortunately, a complete anonymization of RCD that would actually exclude privacy risks comprehensively and thus make privacy law inapplicable is either infeasible for relevant datasets [6] or would largely limit the usefulness of these data [7]. Developing a consent template as well as establishing a workable process for granting consent to establish a legal basis for RCD reuse that is scalable, legally and ethically appropriate, and broadly accepted is thus a crucial prerequisite for the establishment of a learning health care system.

The content of patients' consent largely determines the secondary use options. To allow data use across hospitals and federal states, it is particularly important that essential elements of the patient information and consent forms be standardized. Since not all purposes for which the data might be used in the future can be accurately foreseen at the time of the initial consent, it is necessary to describe more broadly the purpose of the intended data use for medical research ("broad consent").

However, opinions regarding how broadly the purposes of data use may or should be described in a consent differ among experts and authorities for data protection, research ethics, health care, patient representatives, and health insurance [8, 9]. To date, there exists no national broad consent document that has been agreed upon on the basis of a national coordination process involving all the relevant stakeholder groups and authorities - in other European countries covered by the GDPR or internationally.

Controversial debates focus, for example, on

- whether the broad consent approach should be narrower, that is, constrained to certain diseases or areas of medical research [10],

- whether an opt-in or an opt-out model is preferable [11],

- whether a dynamic consent approach should supplement or even completely replace the initial broad consent [12], or

- what the essential information is that any broad consent form should entail [13]. 
The German Medical Informatics Initiative (MII) is a program funded by the German Federal Ministry of Education and Research (BMBF) [14]. Currently, all university hospitals in Germany are participating in this initiative. The aim is to establish a nationwide infrastructure for the secondary use and sharing of health care data and human biosamples to improve medical care and biomedical research [1, 2]. Over the past four years, the "Consent" working group instituted by the National Steering Board of the MII has performed a detailed requirements analysis to guide further development of a consent procedure and text to enable MII implementation. Based on these requirements, the working group then iteratively developed

- broad consent documents including a patient information (PI) and a patient consent (PC) form,

- a guidance document (GD) describing binding rules for implementing the broad consent and accompanying safeguards, and

- four detailed analysis documents on key discussion points.

Based on these developments, in April 2020, all relevant authorities in Germany agreed to a national GDPR-compliant modularized standard for broad consent consisting of the abovementioned broad consent and guidance documents.

The aim of this paper is to describe the steps of the development (Methods section); to outline the key discussion points and corresponding analysis results, which may be relevant for similar discussions internationally; and finally, to present the resulting texts (in English and German).

\section{Methods}

\section{The "Consent" working group}

The "Consent" working group consists of approximately 20 specialists nominated by all four MII consortia [15-18] and the central coordination structure of the MII [14]. Their expertise covers the relevant domains of clinical medicine, medical ethics, law, biobanking, medical informatics, and various domains of biomedical science. From late 2016 through April 2020, the working group met 25 times, with each meeting having a median of 15 participants and lasting an average of 6 hours.

\section{Requirements Analysis}

Initially, we performed a detailed analysis of the conditions for the secondary use of health data and human biosamples from the medical, technical, legal, and ethical points of view. For this purpose, we took the projects of the four participating MII consortia with their specific 
requirements for the type of secondary use [15-18] as a starting point. However, since the infrastructure established within the scope of the MII is also intended to serve usage requests from external researchers [19], we extended the scope of our analysis. In addition, we obtained brief expert opinions to clarify specific, mainly national, legal issues identified during the requirements analysis phase.

\section{Document development and consensus process}

The initial drafts for the broad consent were built on existing German standards for broad consent in biobank research and previously published systematic reviews of existing guidance for broad consent texts [1, 20,21]. Subsequently, the abovementioned core documents were developed for patient information (PI) and patient consent (PC), along with the guidance document (GD) for consent implementation and safeguards.

The drafts were further developed within an iterative consensus process involving all 52 German medical research ethics committees, all 18 German federal and state level data protection authorities, and a consultation group including patient representatives of the Federal Joint Committee (the highest decision-making body of the joint self-government of physicians, dentists, hospitals and health insurance funds in Germany). Constraints and conditions imposed by stakeholders on the broad consent procedure during the consensus process that are not directly relevant to the PI and PC texts were incorporated into the GD, which thus evolved into a binding governance component.

For a more detailed description of the consultation procedures with the German data protection authorities and the German research ethics committees, see textbox 1.

\section{Place textbox 1 ("Consultation with Data Protection Authorities and Research Ethics Committees") here}

\section{Results}

We present the results of the requirements analysis and how these are addressed in the final German standard for broad consent regarding the secondary use of health care data and human biosamples. At the end of the results section, we present our position on additional or alternative consent elements such as dynamic consent. 


\section{Requirements}

The requirements analysis and legal opinions revealed the following key requirements for the consent documents:

a) standardization of texts,

b) broadness of purpose,

c) existence of a guidance document and mandatory safeguards, and

d) modular and expandable structure of the texts.

\section{Standardization of texts}

The need for a uniform consent arises from the consideration that a uniformly applicable legal basis is required for cross-site use. Whether a participating hospital can provide data for a research project should not depend on differences in wording and related uncertainties regarding their interpretation. In addition, the complex and laborious consensus process involving the different stakeholders presented here was only feasible for exactly one text and not the numerous variants that typically arise from site-specific adaptation, as previously observed for the biobanking consent templates that the current developments are based on. In the future, it cannot be ruled out that individual patients will give consent in several of the participating university hospitals. In these cases, however, they should not be surprised or unsettled by unexpected content or new formulations. Finally, some of the overarching safeguards (see below) also require a uniform approach and thus also a uniform patient information and consent declaration. For these reasons, all stakeholders agreed early during the development process that consensus documents should be adopted verbatim at all sites. Only placeholders explicitly provided for local adaptations (e.g., name of the controller) are to be replaced locally in a suitable and strictly prescribed manner.

\section{Broadness of purpose}

A usable infrastructure for secondary use of RCD to facilitate biomedical research and a learning health care system requires a broad definition of purpose. It is impossible to know and describe all meaningful future research goals and evaluation questions at the time patients are asked for their consent. In particular, the unforeseeable development of biomedical research requires the adjustment of research agendas to evolving challenges such as multimorbidity and its associated problems [22, 23]. Clearly, consent limited to single disease areas cannot support urgently needed research to develop new therapeutic approaches for multiple diseases and to detect and prevent the adverse effects of therapy interactions. 
In addition, if we wanted to restrict consent in a disease-specific way, we would first need to know the diagnosis and other underlying conditions. However, this information is often not known at the time of admission to the clinic. A later time of consent, on the other hand, would induce selection bias by systematically excluding patients who have lost the ability to consent due to disease progression or death.

Moreover, the continuous advancement of biomedical knowledge affects disease classification and may reveal connections between diseases that originally appeared to be quite disparate (e.g., [24, 25]). These developments will only be accelerated by the growing toolbox of genetic and epigenetic disease research (e.g., [26]). This evolution of disease classification and description over time will contribute to opacity for data donators and foreseeably lead to legal uncertainties in the use of data and biosamples.

Furthermore, data and biosamples are also needed for methodological research questions, which again cannot always be meaningfully assigned to a specific disease area. Finally, for many research questions, control groups are also needed, i.e., groups of patients who do not have the disease under investigation but who are equally severely ill and have a comparable degree of hospitalization.

\section{Guidance document and mandatory safeguards}

The guidance document first describes the possibilities and limits of adapting the texts to local particularities while staying within the boundaries defined by the MII consensus procedure together with the data protection authorities, research ethics committees, and other stakeholders. Outside the MII, all users of these texts are of course free to adapt them further to their own needs if they do not require the legal and ethical security provided by the explicit consensus procedure. In these cases, however, the MII logo and the reference to the template solution of the MII may no longer be used.

The guidance document further describes the following mandatory safeguards:

A) Independent review of each individual research project using data or biosamples:

- In accordance with the Declaration of Helsinki (No. 23, [27]) of the World Medical Association (WMA) and the complementary WMA guidance in the Declaration of Taipei (No. 19, [28]), each research protocol of any project wishing to use the data and, if applicable, biosamples consented to here must be submitted for review and guidance to an independent research ethics committee. 
- In addition, any use of data and biosamples must be reviewed and approved by the Use \& Access Committee of the respective hospital. In particular, it must be verified that the desired type of data use fits the nature and specific options of the consent. Use \& Access Committees further review the feasibility and scientific quality of secondary use projects.

B) Organizational measures to protect patients from involuntary disclosure of protected information:

- Detailed and nationally harmonized contracts and terms of use bind all users of data and biosamples.

- If feedback on individual (unsolicited/incidental) patient findings is planned or foreseeable, the feedback procedure must be explicitly described and reviewed by a research ethics committee and the Use \& Access Committees.

C) Comprehensive information for patients and public transparency:

- Short films with sound and subtitles in English and German as well as flyers were developed by the MII and should be employed to improve the information provided to patients.

- Every research project that uses data or biosamples is transparently presented on a publicly accessible website referenced in the PI. In addition to a generally comprehensible description of the project's objectives, information on funding sources must also be provided.

- Any patient and any other interested individual can sign up for a newsletter on the transparency website that provides short-term email updates on newly registered research projects before actual data delivery starts. This pre-use transparency regarding the objectives of new projects empowers patients to withdraw consent if they do not wish to further support this type of research. It further empowers third parties to follow the research agenda and signal concerns if necessary.

\section{Modular and expandable approach}

A modular and expandable approach for the consent documents allows participating sites to present to patients only those options that actually make sense locally. For example, a site that does not offer biobanking services does not need to present the consent text module describing the secondary use of biosamples. Modularity also allows further development of new text modules without forcing reconsent to already finalized modules. 
Textbox 2 describes the modules for the initial development phase. Each module consists of a description of the patient information and one or more associated consent option on the informed consent form.

\section{Place textbox 2 ("Text modules of the documents for patient information (PI) and patient consent") here}

\section{Further legal constraints and requirements}

The in-depth assessment of the regulatory constraints involved four legal briefs that clarified the following issues:

A) Particular state legal regulations from selected German states do not contradict a broad consent approach.

B) Anonymization does not have to be absolute according to the GDPR, i.e., it does not have to prevent reidentification for all times and under all circumstances.

C) The acts of informing patients and obtaining consent must be performed by competent and trained staff but not necessarily by physicians.

D) Documentation of consent does not necessarily have to be paper-and-pencil based. Digital forms for obtaining consent are also possible.

E) Medical confidentiality (regulated in Germany via criminal law) and data protection law (regulated EU-wide via public or civil law, with numerous state-level additions and alterations) each represent restrictions on data and biosample use that must be considered separately. Thus, consent to cross-institutional use of data and biosamples must also include a release of confidentiality to cover the criminal law aspect. This release from the obligation of maintaining confidentiality can also be implicit if the consent explicitly states that data and biosamples from the protected treatment relationship are to be released.

\section{Position on additional or alternative consent elements such as dynamic consent}

A core controversy around broad consent is the lack of information at the time of consent about the specific research questions that secondary use projects will address. As described above, the MII broad consent addresses this challenge by establishing various safeguards, including the provision of broad public transparency about new secondary use projects at least one week prior to their start. This allows patients who donated data and, if applicable, biosamples via a broad consent to monitor the specific questions of secondary use projects and to withdraw 
their consent if they do not wish to further support this type of research. This broad transparency, however, explicitly does not provide for informing individual patients about which of the new secondary use projects included their individual data and, if applicable, biosamples. Proponents of the so-called dynamic consent model, however, argue that such broad transparency is insufficient and that one additional feature is required. Each project should require individual (re)consent, resulting in personalized and project-specific transparency (each individual should be informed about what projects require their personal RCD) [12, 29, 30]. It is questionable whether this additional feature substantially improves patients' rights or respect for patient autonomy because in the absence of this additional feature, broad transparency already empowers patients to withdraw their consent in response to projectspecific information [31].

Beyond the uncertainty regarding the improvement of patient autonomy, this additional feature comes with a series of further risks for individual patients while also bearing the potential to negatively impact the validity and relevance of secondary use projects for improving health care. The need for explicit personalized consent prior to every new secondary use project means that all data from patients who can no longer be reached for reconsent will be dropped from future investigations. This would severely limit the informative value of many analyses, as, for example, younger and healthier patients with good disease courses would be significantly overrepresented in the data.

Personalized information about secondary use projects would also entail communication challenges if the data of patients were to be included in a study on the basis of a risk or disease marker found and the patients concerned were informed of the presence of the marker for the first time on a website or via an app when asked for reconsent. Another argument against dynamic consent is that the implementation of a platform that contains sensitive consent information but must also be easily accessible to patients inevitably entails additional data safety and security risks (cf. [32]).

In view of the risks and challenges of a dynamic consent model outlined above and the questionable increase in patient autonomy compared to that afforded by the MII broad consent model, we successfully argued that the additional risks outweighed the potential benefits.

\section{Consensus results, final documents and translations}

The final round of consultation with all the research ethics committees in Germany belonging to the Association of Medical Ethics Committees in Germany ended in June 2019 with only two maintained comments from one ethics committee. The version of the consent documents that was reduced in scope after the final vote by the data protection authorities (e.g., without the possibility of transferring data and, if applicable, biosamples to countries with lower data 
protection than in the EU) was once again brought to the attention of all the ethics committees in April 2020.

In April 2020, we finally obtained unanimous approval of the documents from all the data protection authorities in Germany represented in the DPC. The DPC officially published a press release on this result (https://www.datenschutzkonferenzonline.de/media/pm/20200427 Einwilligungsdokumente der Medizininformatik-Initiative.pdf, in German).

The following core documents are available in English and German for download from the MII website (https://www.medizininformatik-initiative.de/en/template-text-patient-consent-forms):

- Consent Documents, Version 1.6d, including Patient Information (6 pages) and Consent Declaration Form (3 pages) with all modules

- Guidance Document (10 pages)

The Consent Documents are also available in Turkish. Additional materials include a flyer and a video that preinforms and sensitizes potential data donors and the public in general.

\section{Discussion}

The new German standard presented here shows that a GDPR-compliant broad consent for secondary use of health care data and biosamples for (bio)medical research can fulfil all the requirements of research ethics committees and federal- and state-level data protection authorities. After all, this coordination in Germany involved 18 data protection authorities and approximately 50 ethics committees. Stakeholder engagement accompanied the development process and is being further intensified in the current implementation and evaluation phase.

The value of this comprehensive consent work can only be gauged by looking at various reasons for why Germany has so far not implemented widespread secondary use of electronic patient data. Other countries within the European Union (EU), e.g., Denmark and Estonia, or beyond the EU, e.g., Israel, are much further ahead in terms of creating infrastructures and establishing conditions for the secondary use of data from the health care system for research [33]. Hard factors in this regard include the federal structure of the science and health care system as well as the less dynamic system of self-regulation of public health care in Germany. In addition, soft factors such as a traditionally high weighting of data protection [34] and a high level of concern or low enthusiasm with regard to the sharing and innovative use of personal health data [35] must also be taken into account.

A key challenge of aligning consent documents with the various stakeholders was explaining and justifying the decision to use broad consent and the decision against using alternative models such as dynamic consent [12, 29] or meta consent [36, 37]. The factor that convinced 
those initially in favour of other consent models was the commitment to implement specific measures to achieve the greatest possible transparency for patients about what would later happen with the data in general. These measures include, in particular, a central website that lists and explains all studies that are conducted using the patient data. In addition, patients can sign up for a newsletter that provides information about new studies at least one week before they begin. Such "broad and preuse transparency" allows patients to withdraw their broad consent based on project-specific information before actual project implementation without exposing data donors to additional risks generated by the new data processing infrastructures needed for the dynamic consent model or unnecessarily exacerbating selection bias.

Measures going beyond such broad transparency, as typically required by the so-called dynamic consent models, do not, based on our analyses, provide substantial improvement of patient autonomy but introduce important communication challenges, additional data privacy risks, and biased datasets. For all these reasons, wide acceptance of broad consent has emerged internationally in recent years (e.g., [8, 13, 21, 38]). Recent recommendations for implementing a dynamic consent model do not mention any of the risks and challenges outlined in this paper and should be revised accordingly (e.g., [39], p. 215).

The MII partners will implement the broad consent model with the preuse transparency strategy and evaluate its effectiveness and efficiency. In addition to assessing patient and stakeholder engagement, further evaluation is needed to understand the informational needs of the public, the subjective and objective understanding of the consent form, and the overarching preferences of patients and other stakeholders regarding the long-term transition to a learning health care system.

Due to the extended coordination and consensus procedure with the authorities and the resulting critical loss of time for the project, the originally planned consent option for the transfer of data and biosamples to countries with a lower level of data protection than in the EU was eliminated from the final consensus documents since consensus could not be achieved in the available timeframe. Because the requirements analysis identified this consent option as essential for an internationally connectable infrastructure, it must be addressed as part of the next consensus iteration, which is anticipated to begin in 2021. Further clarifications will also address the question of how the consent text can address the secondary use of health data that were collected prior to the date of the initial broad consent.

In German patient information documents, the concept of the pseudonymization of data has become widely accepted over the last twenty years. The basis for this was a simple and comprehensible definition in German data protection law. However, the GDPR introduced a seemingly narrower definition of pseudonymization in Art. 4, although the term itself is obviously used in a broader sense throughout the GDPR, also in accordance with the previous 
German definition [40, p. 174ff]. In the last round of coordination with the data protection authorities, this ambiguity led to a few representatives of the data protection authorities insisting that the term "pseudonymization" be replaced by the term "coding" in the consent documents published here. Further discussion and consultation will reveal which understanding of the term will prevail throughout Europe.

Last but not least, the appropriateness and acceptability of the broad consent approach depends on how effectively and efficiently the ultimate secondary use projects are conducted and reported. The MII working groups on interoperability and data sharing collaborate with the "Consent" working group to continuously monitor and update the national governance for the secondary use of health data.

Regarding future perspectives, the authors hope that the new German standard for broad consent will contribute to a patient-centred and research-enabling culture of secondary data use. Along with viable and broadly supported research infrastructures, this will support a learning health system. While currently largely limited to academic medicine in Germany, the first steps for extending this approach to wider areas of application, including smaller institutions and private practices without direct interest in supporting research, are currently under consideration. The German standard also intends to cover secondary use of data from clinical registries or even investigator-initiated clinical trials (IITs); a corresponding module is currently under development.

The coronavirus pandemic has shown that it is not easy to quickly and extensively collect data from patients and appropriate controls without time-consuming coordination of the consent forms and procedures with research ethics committees and data protection officers [41-43]. In the future, even acute research questions could be answered more quickly and efficiently than before by using the standardized consent infrastructure described here.

It would be desirable to inform subsequent patients about the risks and benefits of providing data for research before they are admitted to the hospital. However, this would require information campaigns that the MII cannot manage on its own. If such campaigns are successful and trigger a broad social debate about the advantages and disadvantages of making data available for research, consideration could also be given to a legal basis for data use, which should then in any case provide for the possibility of an opt-out for citizens [44].

\section{Acknowledgement}

The MII as a whole is funded by the German Ministry of Education and Research. The authors received the following grants:

Sven Zenker: 01ZZ1602C, 01ZZ1803Q

Page 13 of 21 
Daniel Strech: 01ZZ1605B, 01ZZ1802C

Kristina Ihrig: 01ZZ1606E, 01ZZ1801C

Roland Jahns: 01ZZ1802W

Gabriele Müller: 01ZZ1608D, 01ZZ1801L

Christoph Schickhardt, Eva Winkler: 01ZZ1802A

Georg Schmidt: 01ZZ1603A, 01ZZ1804A

Ronald Speer: 01ZZ1609A, 01ZZ1803A

Johannes Drepper: 01ZZ1601, 01ZZ1805

\section{References}

1. Safran C, Bloomrosen M, Hammond WE, Labkoff S, Markel-Fox S, Tang PC, et al. Toward a national framework for the secondary use of health data: an American Medical Informatics Association White Paper. J Am Med Inform Assoc. 2007;14(1):1-9. Epub 2006/11/02. doi: 10.1197/jamia.M2273. PubMed PMID: 17077452; PubMed Central PMCID: PMCPMC2329823.

2. Luo J, Wu M, Gopukumar D, Zhao Y. Big Data Application in Biomedical Research and Health Care: A Literature Review. Biomed Inform Insights. 2016;8:1-10. Epub 2016/02/05. doi: 10.4137/BII.S31559. PubMed PMID: 26843812; PubMed Central PMCID: PMCPMC4720168.

3. Murdoch TB, Detsky AS. The inevitable application of big data to health care. JAMA. 2013;309(13):1351-2. Epub 2013/04/04. doi: 10.1001/jama.2013.393. PubMed PMID: 23549579.

4. Chambers DA, Feero WG, Khoury MJ. Convergence of Implementation Science, Precision Medicine, and the Learning Health Care System: A New Model for Biomedical Research. JAMA. 2016;315(18):1941-2. Epub 2016/05/11. doi: 10.1001/jama.2016.3867. PubMed PMID: 27163980; PubMed Central PMCID: PMCPMC5624312.

5. Faden RR, Kass NE, Goodman SN, Pronovost P, Tunis S, Beauchamp TL. An ethics framework for a learning health care system: a departure from traditional research ethics and clinical ethics. Hastings Cent Rep. 2013;Spec No:S16-27. Epub 2013/02/27. doi: 10.1002/hast.134. PubMed PMID: 23315888.

6. Ohm P. Broken Promises of Privacy: Responding to the Surprising Failure of Anonymization. UCLA Law Review. 2010;57:1701-77.

7. El Emam K, Álvarez C. A critical appraisal of the Article 29 Working Party Opinion 05/2014 on data anonymization techniques. International Data Privacy Law. 2015;5(1):73-87. doi: 10.1093/idpl/ipu033. 
8. Garrison NA, Sathe NA, Antommaria AH, Holm IA, Sanderson SC, Smith ME, et al. A systematic literature review of individuals' perspectives on broad consent and data sharing in the United States. Genet Med. 2016;18(7):663-71. Epub 2015/11/20. doi: 10.1038/gim.2015.138. PubMed PMID: 26583683; PubMed Central PMCID: PMC4873460.

9. Hirschberg I, Kahrass H, Strech D. International requirements for consent in biobank research: qualitative review of research guidelines. J Med Genet. 2014;51(12):773-81. Epub 2014/10/30. doi: 10.1136/jmedgenet-2014-102692. PubMed PMID: 25351952.

10. Ewing AT, Erby LA, Bollinger J, Tetteyfio E, Ricks-Santi LJ, Kaufman D. Demographic differences in willingness to provide broad and narrow consent for biobank research. Biopreserv Biobank. 2015;13(2):98-106. Epub 2015/04/01. doi: 10.1089/bio.2014.0032. PubMed PMID: 25825819; PubMed Central PMCID: PMCPMC4574731.

11. McCall C. Opt-out digital health records cause debate in Australia. Lancet. 2018;392(10145):372. Epub 2018/08/29. doi: 10.1016/S0140-6736(18)31726-4. PubMed PMID: 30152368.

12. Williams H, Spencer K, Sanders C, Lund D, Whitley EA, Kaye J, et al. Dynamic consent: a possible solution to improve patient confidence and trust in how electronic patient records are used in medical research. JMIR Med Inform. 2015;3(1):e3. Epub 2015/01/15. doi: 10.2196/medinform.3525. PubMed PMID: 25586934; PubMed Central PMCID: PMC4319083.

13. Grady C, Eckstein L, Berkman B, Brock D, Cook-Deegan R, Fullerton SM, et al. Broad Consent for Research With Biological Samples: Workshop Conclusions. Am J Bioeth. 2015;15(9):34-42. Epub 2015/08/26. doi: 10.1080/15265161.2015.1062162. PubMed PMID: 26305750; PubMed Central PMCID: PMC4791589.

14. Semler SC, Wissing F, Heyder R. German Medical Informatics Initiative. A National Approach to Integrating Health Data from Patient Care and Medical Research. Methods Inf Med. 2018;57(S 01):e50-e6. doi: 10.3414/ME18-03-0003. PubMed PMID: 30016818.

15. Haarbrandt B, Schreiweis B, Rey S, Sax U, Scheithauer S, Rienhoff O, et al. HiGHmed - An Open Platform Approach to Enhance Care and Research across Institutional Boundaries. Methods Inf Med. 2018;57(S 01):e66-e81. doi: 10.3414/ME18-02-0002. PubMed PMID: 30016813.

16. Prasser F, Kohlbacher O, Mansmann U, Bauer B, Kuhn KA. Data Integration for Future Medicine (DIFUTURE). Methods Inf Med. 2018;57(S 01):e57-e65. doi: 10.3414/ME1702-0022. PubMed PMID: 30016812.

17. Prokosch HU, Acker T, Bernarding J, Binder H, Boeker M, Boerries M, et al. MIRACUM: Medical Informatics in Research and Care in University Medicine. Methods 
Inf Med. 2018;57(S 01):e82-e91. doi: 10.3414/ME17-02-0025. PubMed PMID: 30016814

18. Winter A, Stäubert S, Ammon D, Aiche S, Beyan O, Bischoff V, et al. Smart Medical Information Technology for Healthcare (SMITH). Methods Inf Med. 2018;57(S 01):e92e105. doi: 10.3414/ME18-02-0004. PubMed PMID: 30016815.

19. Gehring S, Eulenfeld R. German Medical Informatics Initiative: Unlocking Data for Research and Health Care. Methods Inf Med. 2018;57(S 01):e46-e9. doi: 10.3414/ME18-13-0001. PubMed PMID: 30016817.

20. Strech D, Kahrass H, Hirschberg I. Research Guideline Recommendations for Broad Consent Forms in Biobank Research and How They Are Currently Addressed in Practice. Am J Bioeth. 2015;15(9):60-3. Epub 2015/08/26. doi: 10.1080/15265161.2015.1062169. PubMed PMID: 26305759.

21. Strech D, Bein S, Brumhard M, Eisenmenger W, Glinicke C, Herbst T, et al. A template for broad consent in biobank research. Results and explanation of an evidence and consensus-based development process. Eur J Med Genet. 2016;59(6-7):295-309. Epub 2016/05/01. doi: 10.1016/j.ejmg.2016.04.002. PubMed PMID: 27130428.

22. Bierman AS, Tinetti ME. Precision medicine to precision care: managing multimorbidity. Lancet. 2016;388(10061):2721-3. Epub 2016/12/08. doi: 10.1016/S01406736(16)32232-2. PubMed PMID: 27924764.

23. Barnett K, Mercer SW, Norbury M, Watt G, Wyke S, Guthrie B. Epidemiology of multimorbidity and implications for health care, research, and medical education: a cross-sectional study. Lancet. 2012;380(9836):37-43. Epub 2012/05/15. doi: 10.1016/S0140-6736(12)60240-2. PubMed PMID: 22579043.

24. Pagano G, Polychronis S, Wilson H, Giordano B, Ferrara N, Niccolini F, et al. Diabetes mellitus and Parkinson disease. Neurology. 2018. Epub 2018/04/08. doi: 10.1212/WNL.0000000000005475. PubMed PMID: 29626177.

25. Gelfand JM, Wan MT. Psoriasis: a novel risk factor for type 2 diabetes. Lancet Diabetes Endocrinol. 2018. Epub 2018/05/26. doi: 10.1016/S2213-8587(18)30127-X. PubMed PMID: 29799414.

26. Brainstorm-Consortium. Analysis of shared heritability in common disorders of the brain. Science. 2018;360(6395). Epub 2018/06/23. doi: 10.1126/science.aap8757. PubMed PMID: 29930110.

27. WMA. World medical association declaration of helsinki: Ethical principles for medical research involving human subjects. Journal of the American Medical Association: World Medical Association, 2013 [cited 2019-01-25]. Doc. 20. Available from: http://dx.doi.org/10.1001/jama.2013.281053 
28. WMA. WMA Declaration of Taipei on Ethical Considerations regarding Health Databases and Biobanks. Taipei: World Medical Association, 2016 [cited 2019-01-25]. Available from: https://www.wma.net/policies-post/wma-declaration-of-taipei-on-ethicalconsiderations-regarding-health-databases-and-biobanks/

29. Kaye J, Whitley EA, Lund D, Morrison M, Teare H, Melham K. Dynamic consent: a patient interface for twenty-first century research networks. Eur J Hum Genet. 2015;23(2):141-6. Epub 2014/05/08. doi: 10.1038/ejhg.2014.71. PubMed PMID: 24801761 ; PubMed Central PMCID: PMC4130658.

30. Budin-Ljøsne I, Teare HJ, Kaye J, Beck S, Bentzen HB, Caenazzo L, et al. Dynamic Consent: a potential solution to some of the challenges of modern biomedical research. BMC Med Ethics. 2017;18(1):4. Epub 2017/01/27. doi: 10.1186/s12910-016-0162-9. PubMed PMID: 28122615; PubMed Central PMCID: PMC5264333.

31. Steinsbekk KS, Kare Myskja B, Solberg B. Broad consent versus dynamic consent in biobank research: Is passive participation an ethical problem? European Journal of Human Genetics. 2013;21(9):897 - 902. doi: 10.1038/ejhg.2012.282.

32. Thiel DB, Platt J, Platt T, King SB, Fisher N, Shelton R, et al. Testing an online, dynamic consent portal for large population biobank research. Public Health Genomics. 2015;18(1):26-39. Epub 2014/11/02. doi: 10.1159/000366128. PubMed PMID: 25359560; PubMed Central PMCID: PMC4289420.

33. Bertelsmann-Stiftung. \#SmartHealthSystems. Digitalisierungsstrategien im internationalen Vergleich. Gütersloh: Bertelsmann Stiftung, 2018 [cited 2018-11-29]. Doc. November 2018. Available from: https://www.bertelsmannstiftung.de/fileadmin/files/Projekte/Der_digitale_Patient/VV_SHS-Gesamtstudie_dt.pdf

34. Custers B, Dechesne F, Sears AM, Tani T, van der Hof S. A comparison of data protection legislation and policies across the EU. Computer Law \& Security Review. 2018;34(2):234-43. doi: 10.1016/j.clsr.2017.09.001.

35. Voigt TH, Holtz V, Niemiec E, Howard HC, Middleton A, Prainsack B. Willingness to donate genomic and other medical data: results from Germany. Eur J Hum Genet. 2020;28(8):1000-9. Epub 2020/04/03. doi: 10.1038/s41431-020-0611-2. PubMed PMID: 32238912; PubMed Central PMCID: PMCPMC7381614.

36. Ploug T, Holm S. Meta Consent - A Flexible Solution to the Problem of Secondary Use of Health Data. Bioethics. 2016;30(9):721-32. Epub 2016/10/22. doi: 10.1111/bioe.12286. PubMed PMID: 27628305; PubMed Central PMCID: PMC5108479.

37. Ploug T, Holm S. Meta consent: a flexible and autonomous way of obtaining informed consent for secondary research. BMJ. 2015;350:h2146. Epub 2015/05/09. doi: 10.1136/bmj.h2146. PubMed PMID: 25952952. 
38. Jahns R, Geiger J, Schlünder I, Strech D, Brumhard M, Graf von Kielmansegg S. Broad donor consent for human biobanks in Germany and Europe: a strategy to facilitate crossborder sharing and exchange of human biological materials and related data. JLabMed. 2019;43(6):291-9. doi: https://doi.org/10.1515/labmed-2017-0064.

39. WHO. Digital Health Platform Handbook: Building a Digital Information Infrastructure (Infostructure) for Health. Geneva: World Health Organization and International Telecommunication Union, 2020 [cited 2021-04-23]. Available from: https://apps.who.int/iris/handle/10665/337449

40. Dierks C, Roßnagel A. Sekundärnutzung von Sozial- und Gesundheitsdaten Rechtliche Rahmenbedingungen. Berlin: MWV; 2019.

41. Elwyn G, Seagrove A, Thorne K, Cheung WY. Ethics and research governance in a multicentre study: add 150 days to your study protocol. BMJ. 2005;330(7495):847. Epub 2005/04/09. doi: 10.1136/bmj.330.7495.847. PubMed PMID: 15817562; PubMed Central PMCID: PMCPMC556088.

42. Snooks $H$, Hutchings $H$, Seagrove A, Stewart-Brown S, Williams J, Russell I. Bureaucracy stifles medical research in Britain: a tale of three trials. BMC Med Res Methodol. 2012;12:122. Epub 2012/08/18. doi: 10.1186/1471-2288-12-122. PubMed PMID: 22898336; PubMed Central PMCID: PMCPMC3537588.

43. Eichler M, Schmitt J, Schuler MK. Die Dauer von Ethikvoten in Deutschland - am Beispiel einer nicht-interventionellen Beobachtungsstudie mit 44 teilnehmenden Zentren (PROSa). ZEFQ. 2019;146:15-20. Epub 2019/09/02. doi: 10.1016/j.zefq.2019.07.006. PubMed PMID: 31473073.

44. Strech D, Graf von Kielmansegg S, Zenker S, Krawczak M, Semler SC. Wissenschaftliches Gutachten: „Datenspende“ - Bedarf für die Forschung, ethische Bewertung, rechtliche, informationstechnologische und organisatorische Rahmenbedingungen. Erstellt für das Bundesministerium für Gesundheit Berlin: Bundesministerium für Gesundheit, 2020 [cited 2020-12-15]. Doc. Version 1.1. Available from: https://www.bundesgesundheitsministerium.de/fileadmin/Dateien/5_Publikationen/Minis terium/Berichte/Gutachten_Datenspende.pdf 


\section{Textboxes}

\section{Textbox 1: Consultation with Data Protection Authorities and Research Ethics Committees}

The consultation of the MII "Consent" working group with data protection authorities in Germany was initiated by a presentation and discussion of core requirements and an initial draft consent with the representatives of two working groups of the Conference of Independent Federal and State Data Protection Authorities (Data Protection Conference, DPC). After reaching initial agreement on the draft, we consulted all the research ethics committees in Germany represented in the Association of Medical Ethics Committees in Germany (https://www.akek.de/en/). This consultation was mediated by the "Biobanking" working group within the association, which had previously drafted and coordinated a broad consent text for biobank research [38]. The texts, which were improved by extensive feedback from the ethics committees, were then again submitted to the previously involved working groups of the DPC. However, previously accepted formulations and basic assumptions had since been rejected by the data protection authorities, making a new, lengthy consultation necessary. In the end, only the involvement of the authority heads as well as a series of compromises and the waiver of the possibility of transferring data and samples to third countries (countries with a lower level of data protection than in the EU) made an overarching consensus possible.

Approximately 150 written comments from different stakeholder groups were submitted during the revision process. Some of the comments contradicted each other. It was therefore impossible to implement all requested changes completely. Through a structured change management process, the working group ensured that each comment was explicitly evaluated, discussed, and decided upon with respect to its relevance and addressability. All stakeholders received detailed responses to each comment, either in the form of a description of how it had been considered or implemented or with a comprehensive explanation of why a comment had not led to an adjustment of the texts.

The detailed responses to the more fundamental questions and comments of the data protection authorities resulted in four position papers, which were made publicly available as German-language documents (https://www.medizininformatikinitiative.de/de/mustertext-zur-patienteneinwilligung). These documents address, 
among other things, the rationale for broad consent, the issues and challenges associated with dynamic consent, possible types of research transparency, and the need for cooperation on data sharing with non-EU countries.

The final consensus documents as well as the interim results were also published online in a timely fashion to maximize transparency after the MII internal quality assurance and review was completed.

\section{Textbox 2: Text modules of the documents for patient information (PI) and patient consent (PC)}

\begin{tabular}{|c|c|}
\hline Modules & Explanation \\
\hline $\begin{array}{l}\text { Health Care Data and general } \\
\text { procedures } \\
\text { (mandatory information for } \\
\text { each broad consent text) }\end{array}$ & $\begin{array}{l}\text { The patient information describes relevant sources of } \\
\text { routine health care data, the purpose of secondary use, the } \\
\text { principle of non-interference with individual treatment/care, } \\
\text { the risks associated with secondary use, and the } \\
\text { constraints regarding international data sharing. }\end{array}$ \\
\hline $\begin{array}{l}\text { Biosamples including limited } \\
\text { additional samples } \\
\text { (optional with separate } \\
\text { consent option) }\end{array}$ & $\begin{array}{l}\text { The patient information explains that human tissue } \\
\text { specimens and/or body fluids taken for diagnosis/treatment } \\
\text { that (after conclusion of the examination or treatment) are } \\
\text { no longer needed can be useful for medical research. The } \\
\text { text module also offers an option to donate additional } \\
\text { biosamples for research (e.g., a limited amount of blood } \\
\text { collected during routine blood sampling). }\end{array}$ \\
\hline $\begin{array}{l}\text { Health Insurance Data } \\
\text { (optional with separate } \\
\text { consent option) }\end{array}$ & $\begin{array}{l}\text { The patient information describes access to and linking of } \\
\text { patient data with patient-related data from health insurers. } \\
\text { The text module offers the option of consenting to both } \\
\text { retrospective and prospective use of health insurance data, } \\
\text { such as medical and nursing services used or medicine } \\
\text { prescribed. }\end{array}$ \\
\hline
\end{tabular}




\begin{tabular}{|c|c|}
\hline $\begin{array}{l}\text { Recontacting } \\
\text { (mandatory with separate } \\
\text { consent option) }\end{array}$ & $\begin{array}{l}\text { The patient information offers the patient a choice to give } \\
\text { permission to be recontacted to collect further information } \\
\text { or even to provide feedback on research results related to } \\
\text { the individual patient and explains the risks associated with } \\
\text { such communication. In addition, we point out that in rare } \\
\text { cases, feedback in the form of analysis results with very } \\
\text { high individual relevance may be provided by the treating } \\
\text { physician even without explicit consent based on legal or } \\
\text { ethical obligations. }\end{array}$ \\
\hline
\end{tabular}

